\title{
Monitoring metabolic activities of small animals by means of microcalorimetry
}

\author{
Ingolf Lamprecht
}

Institute for Biophysics, Free University Berlin

Thielallee 63, D-14195 Berlin, Germany

\begin{abstract}
The present paper deals with direct and indirect calorimetric experiments on smaller terrestrial and aquatic animals in the range of $\mathrm{mg}$ and $\mathrm{g}$ wet weight. Especially, the energy metabolism during development and growth, under decrease of oxygen concentration (hypoxia/anoxia) and with adverse or toxic environmental conditions was studied.

The results demonstrate the broad versability of direct calorimetry as a sensitive, nonspecific and non-invasive method for investigations of living organisms.
\end{abstract}

\section{INTRODUCTION}

Since the early beginning of Crawford (1788) and Lavoisier (1780) thermal analysis has always included biological calorimetry (ref.1). Besides microorganisms terrestrial animals were calorimetrically investigated covering insects, amphibia, reptiles up to domestic animals and man (ref.2). Plants found attention only in recent years due to their usually low metabolism, combined with a large surface/volume ratio and the troubling contribution of evaporation (ref.3). In comparison to terrestrial animals much less papers are found in the literature about direct calorimetry on aquatic systems supposedly due to the large heat capacity of the surrounding water which enhances the thermal inertia of the calorimeter. Last not least further experimental difficulties result from a nonsufficient oxygen supply to the organisms (ref.4).

Again Lavoisier was the first to apply indirect calorimetry as an additional information on the general metabolism of an animal under research or even as an easier surrogate for true direct calorimetric investigations. Indirect calorimetry in the classical sense means measuring the oxygen consumption and carbon dioxide production, determination of the respiratory quotient and by use of data from combustion energy the calculation of the real heat production. Heat flows in animals or through whole ecosystems were frequently calculated by applying Hess' law to data obtained by bomb calorimetry (ref.5,6). In modern times isotope dilution techniques, determination of the heart rate or of oxygen concentrations in venous and arterial blood were included as indirect calorimetric methods (ref.5).

Among the small "calorimeter sized" animals terrestrial insects are the most frequently investigated ones since they are easy to obtain, to breed, to keep and to handle. At heat production rates of 10 to $100 \mathrm{~mW} / \mathrm{g}$ wet weight (ww) and masses between 100 and $1000 \mathrm{mg}$ one expects calorimetric signals of 1 to $10 \mathrm{mV}$ which pose no great demands on the sensitivity of the instrument and the stability of the baseline.

Development and growth was followed calorimetrically in several insects, among them the meal worm Tenebrio molitor L., the cockroach Blattella germanica L. and the wax moth Galleria mellonella (ref.710), while other insects were included in experiments on the influence of xenobiotics, e.g. in forest ecosystems (ref.11-13). Social insects like honeybees, bumblebees and hornets were reviewed recently (ref.14). Terrestrial organisms are more easy to monitor calorimetrically since only minor problems arise with evaporation and the large thermal inertia due to water in the vessel. Nevertheless, several aquatic animals were studied during the last two decades, mainly connected with stress situations occuring in their habitats. During experiments on the host-parasite relationship between the water snail Biomphalaria glabrata and the pair worm Schistosoma mansoni (ref.15) a combination of endoscopy and calorimetry was developed to 
monitor the locomotor activities of animals and to compare them with patterns found in the calorimetric signal (ref.16). This system was adapted later on and connected with an infrared vision device for other investigations (ref.17).

The present paper concentrates on experiments with smaller animals between some $10 \mathrm{mg}$ and a few gram wet weight that fit into commercially available Calvet type microcalorimeters of 15 or $100 \mathrm{ml}$ active volume or into LKB flow through systems of a few milliliter. Both, terrestrial and aquatic animals are presented.

\section{DEVELOPMENT AND GROWTH}

Experiments on the meal worm Tenebrio molitor and the German cockroach Blattella germanica were performed to prove a thermodynamic theory of Zotin (ref.18) about development and aging (ref.7,8) in the light of entropy production rates. According to his theory the bound dissipation function $\Psi_{u}$ defined as difference between the (total) specific dissipation function $\Psi$ and the external dissipation function $\Psi_{\mathrm{d}}$ should approach zero during development and aging.

$$
\Psi_{\mathrm{u}}=\Psi-\Psi_{\mathrm{d}}
$$

$\Psi$ defined by the respirative metabolism of the animal can be determined by usual manometric (Warburg) methods, $\Psi_{\mathrm{d}}$ calorimetrically as it is defined by the heat production. The experiments showed maximum values for $\Psi$ of about $28 \mathrm{~mW} / \mathrm{g}$ and about $15 \mathrm{~mW} / \mathrm{g}$ for $\Psi_{\mathrm{d}}$, both decreasing to approximately $2 \mathrm{~mW} / \mathrm{g}$ at the end of the larval and during the pupal stage. At the same time their difference $\Psi_{u}$ - the bound dissipation function - dropped from a maximum of $13 \mathrm{~mW} / \mathrm{g}$ around day 20 to the predicted value $0 \mathrm{~mW} / \mathrm{g}$ in the pupal stage. With the emergence of the imago after 100 days a thermodynamically "new" organism was born and all three dissipation functions increased again with a consecutive reduction to quite low values (Fig. 1). These data presented for Tenebrio (ref.7,8) are confirmed by those of the German cockroach Blattella germanica (ref.8). The distinct oscillations are partly due to calculating the difference between two small terms. In summary, the calorimetric experiments show that (i) development and growth of these insects tend towards a state of minimum entropy production and highest efficiency and (ii) that this approach occurs in agreement with the predictions derived from the irreversible thermodynamics of open systems (ref.18).

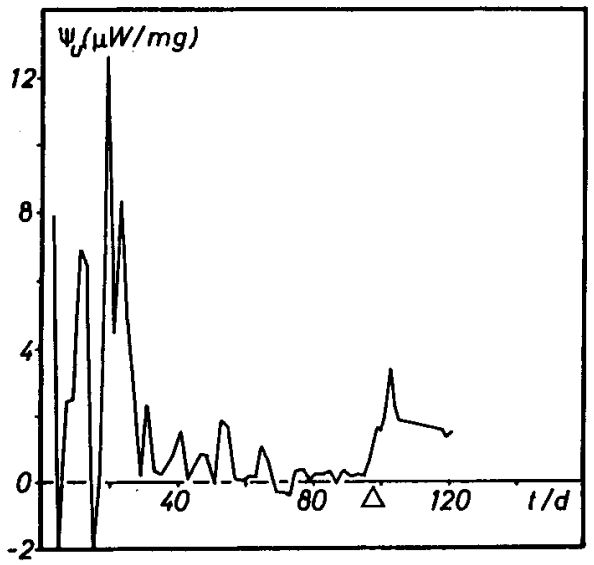

Fig. 1 Bound dissipation function $\Psi_{u}$ depending on the developmental time of the meal worm Tenebrio molitor. The triangle indicates the emergence of the adult. (ref.8)

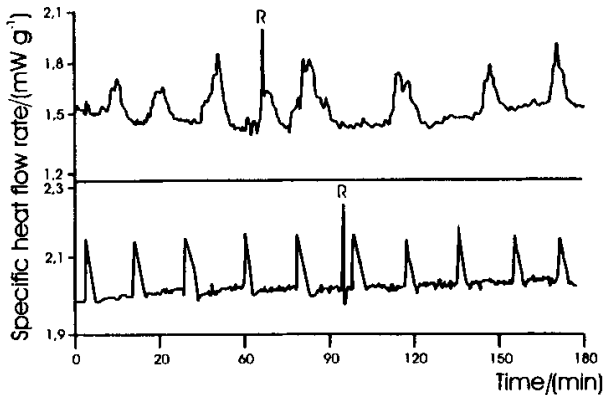

Fig. 2 Calorimetric determinations of periodic muscular activities in Galleria mellonella (above) and Tenebrio molitor (below). "R" indicates rotating movements. (ref.10)

Further calorimetric experiments were performed on the developmental growth of Tenebrio molitor pupae under the influence of the growth regulating and mild toxic effect of a plant extract (ref.19). Morphogenetic failures and prolongation of the periods between moulting were observed calorimetrically as well as transformations in the behaviour. Bouts of rhythmic pupae abdominal movements and bursts of carbon dioxide release from the tracheae were clearly distinguished in the heat flow curves with significant differen- 
ces between the control and the poisoned individuals. Moulting was preceded by an increase in metabolic and locomotor activities and a sudden drop in the calorimetric signal due to evaporation of liberated exuvial fluid. Between two perfect ecdyses (moultings) of the pupae a U-shaped decrease and increase of heat production could be observed as described by other authors and for other organisms, also.

Similar periodic bursts of the heat production rate were seen in calorimetric curves of untreated specimens of the German cockroach Blattella germanica, specially during night, with a sudden increase of $70 \%$ over the level of basal metabolism (ref.8). These bursts of about $5 \mathrm{~min}$ length appeared at intervals of 1 to 3 hours. They were explained as spontaneous paroxism, i.e. consumption of stored oxygen and sudden release of carbon dioxide from the trachaea. Recently, similar phenomena were seen in the calorimetric signals of overwintering hornet queens with frequencies of about 1 per hour (ref.20).

For further investigations on the observed periodic signals in the heat flow curves simultaneous respirometric and calorimetric measurements were performed during the pupal development of the wax moth Galleria mellonella and the mealworm Tenebrio molitor (ref.10). A clear correlation with periodic carbon dioxide release and rhythmic body motions could be established. More irregular structures were found in the wax moth pupae while Tenebrio pupae showed the strongest muscular contractions at the beginning of a ventilation bout and a return to a flat base line afterwards (Fig. 2). Rotating movements of the abdomen in addition to the up-down motions occured more frequently in Galleria pupae and could be clearly distinguished from the latter ones in the calorimetric traces. Such muscular activities act as tracheal ventilation, accelerate the hemolymph circulation and presumably support the heartbeat. Their energetic cost is negligible small with about $1 \%$ of the total heat output showing an elaborated energy use in the pupal stage when animals live on the stored fat reserves.

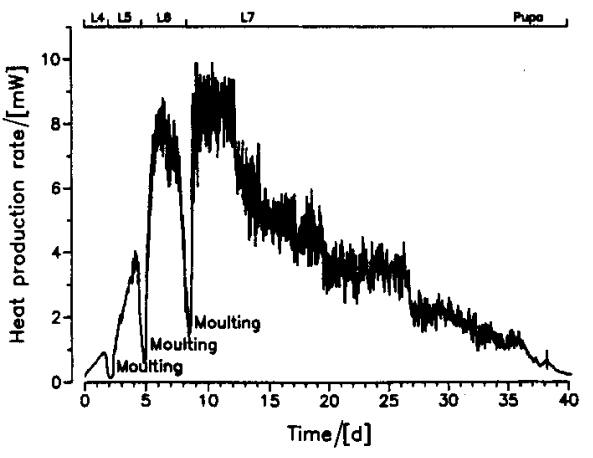

Fig. 3 Power-time curve of the developmental period of a wax moth larva (Galleria mellonella) from the $\mathrm{L} 4$ stage to the pupal stage. The strong endothermic troughs are connected with moulting. The oscillations in the calorimetric signal are produced by locomotor activities of the larva and vanish to the pupal stage. (ref.10)

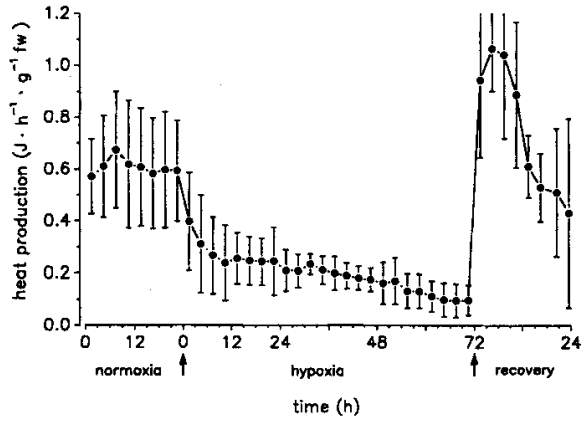

Fig. 4 Power-time curve of the medicinal leech (Hirudo medicinalis) under normoxic and artifical hypoxic conditions and during the subsequent recovery when the change from air to nitrogen (first arrow) is reversed (second arrow). Note the strong aerobic overshoot during recovery.

Short-term calorimetric experiments $(5 \mathrm{~h})$ of individual metabolism in different larval stages and long-term investigations ( $>40 \mathrm{~d}$ ) of development from eggs through all larval stages to pupation were performed on the wax moth Galleria mellonella which is a parasite in weak honeybee colonies (ref.9). They exhibited a rapid temperature increase from 32 to $40^{\circ} \mathrm{C}$ at ambient $28^{\circ} \mathrm{C}$ after a few days in culture. During their development heat production rate grew at a higher rate than mass so that the mass specific heat production rate in larval stages L2/L3 showed a 3.2fold augmentation to stages L4/L5 with values around $160 \mathrm{~mW} / \mathrm{g}$ ww which are near to those of flying insects. As seen in Fig. 2 there is a significant reduction in the following days till pupation. Moreover, one observes strong locomotor activities of all larval stages but nearly none during pupation. The effects of moulting are clearly indicated in Fig. 3 with metabolic reductions down to 7 to $20 \%$ of the preceding value and a recover to the same level half a day later. Long-term experiments on the developmental course of other holometabolous insects such as Tenebrio molitor (ref.7,8) render similar results and offer the chance to establish energy balances for growth from eggs up to the adult insects (ref.8). 


\section{AEROBIC/ANAEROBIC METABOLISM}

Animals rely on aerobic metabolism when conditions of full oxygen concentration of about $21 \%$ exist in air or air saturated water, since respiration renders the highest energy output per mole of consumed substrate. Under normal environmental conditions mild hypoxia with a slightly reduced oxygen tension may arise, but even severe hypoxia down to a complete anoxia can be met, mainly in aquatic systems. Under such circumstances animals have to change more or less to anaerobic pathways with a moderate energy output and other metabolic endproducts. From a physiological as well as from an environmental aspect it is of interest to study such conditions and to determine how animals handle the often toxic situation introduced by oxygen deficiencies.

Though terrestrial animals seldom experience hypoxic or anoxic environmental conditions a few examples of experiments under artificially induced hypoxia or anoxia are found in the literature. The common frog Rana temporaria lowered its heat production rate to around $25 \%$ of the normoxic control under strict anoxia, while under graded hypoxia the rate decreased according to the oxygen concentration. All frogs recovered after hypoxia and anoxia within a few hours (ref.21). Besides the frog two insect species, the migratory locust Locusta migratoria and the moth Manduca sexta, were investigated calorimetrically for the energetic reduction under full anoxia, the time of surviving anoxia and the recovery under normal air. The moth could even withstand anoxic periods of more than $20 \mathrm{~h}$ (ref.22).

The medical leech Hirudo medicinalis was investigated by direct and indirect calorimetry to determine the differences between self-induced (hypercapnic) and artificially induced (hypocapnic) hypoxia (ref.23). Indirect calorimetry was performed by parallel respirometric measurements and biochemical analysis of known anaerobic substrates and endproducts of leech metabolism under self-induced anaerobiosis. When this limnic annelid was kept hypercapnic for longer times under strongly reduced oxygen concentrations heat dissipation dropped to around $13 \%$ and ATP production to $30 \%$ of their normoxic values. This shows that metabolic depression is an important mechanism for the leech to endure long-term anaerobiosis. In the first $\mathbf{8 ~} \mathrm{h}$ of hypoxia there was a full agreement between the measured and the calculated heat dissipation, while for the last period (up to $72 \mathrm{~h}$ ) a larger discrepancy appeared. Only $71 \%$ of the direct calorimetric signal could be explained by the biochemical analysis, so that a further, still unknown anaerobic endproduct has to be assumed. After the end of the artificially induced hypoxic phase air was again bubbled through the surrounding water and the leech quickly recovered with a rapid increase in heat production and oxygen consumption (aerobic overshoot). The heat dissipation rate rose to the double of that at aerobic conditions and returned to the mean normoxic value within 1 day. The whole experiment with all three phases of normoxia, artificially induced hypoxia and recovery is shown in Fig. 4.

All degrees of hypoxia down to strict anoxia are often found in aquatic systems due to a strong microbial oxygen consumption and a lack of motion and ventilation in deeper water layers. Some fish and many other aquatic animals are able to cope with such adverse conditions in their natural habitat. Goldfish (Carassius auratus L.) and tilapia (Oreochromis mossambicus Peters) were investigated as largest objects among the small animals of this review in a 1 litre flow through calorimeter at the Gorlaeus Laboratories in Leiden/Netherlands at various oxygen concentrations (ref.24-26). Carassius answered with a metabolic depression on hypoxia including a strong increase in anaerobic heat production rate at anoxia. The total heat output under anoxia dropped to about $25 \%$ of the normoxic value in accordance with former experiments and biochemical calculations. In investigations on tilapia an additional acidification from $\mathrm{pH} 7.6$ to $\mathrm{pH} 4.0$ had no significant effects on heat production rate during normoxic and hypoxic conditions down to $5 \%$ of air saturation so that hypoxia plus acidification renders no synergistic action on this fish. Tilapia survived severe hypoxia due to metabolic depression (ref.26). When goldfish was used as an aquatic animal model and 2,4-dinitrophenol as a chemical model for environmental pollution and additional stress besides hypoxia, the calorimetric signal increased significantly by more than $50 \%$ under normoxic control conditions although no change in behaviour, locomotor activity and ventilation frequency could be detected. But under hypoxia and anoxia 2,4-dinitrophenol had nearly no influence on the heat flow while the postanoxic heat dissipation was distinctly enhanced compared to the standard rate (ref.27).

During the stagnation phase of freshwater lakes in summer and early autumn the oxygen concentration near the bottom decreases dramatically and sulphide concentrations increase to levels toxic to most animals. Sulphide is known to bind to an essential respiration enzyme (cytochrome c oxidase) resulting in the inhibition of aerobic respiration, the main energy producing pathway of organisms. Therefore, two annelids were investigated which are frequently found in heavily polluted lakes and rivers. They were exposed to varying degrees of hypoxia and sulphide concentrations. While Limnodrilus hoffmeisteri answered with a reduced heat production rate, Tubifex tubifex showed a significantly enhanced heat dissipation under the same conditions. The difference between these two annelids is due to the fact that T.tubifex owns a detoxification system which prevents an aerobic inhibition (ref.28). 
Further investigations on the combination of sulphide and hypoxia were performed in the brackish water polychaete Marenzelleria viridis which inhabits shallow coastal water inlets in the Baltic sea (ref.29). A high concentration of sulphide is found in the sediment of this habitat due to polytrophic conditions. The combination of sulphide and hypoxia strongly increased the heat production rate of adult polychaetes, while larvae acted correspondingly only if the oxygen concentration was high enough. It may be supposed that detoxification processes in the animal are responsible for the elevated energy turnover.

Simultaneous direct and indirect calorimetry under varying conditions of temperature, salinity and hypoxia were performed on the same organism in different developmental stages. Larvae of $M$. viridis did not change their aerobic metabolic activity even at very low oxygen concentrations while the adults reduced their rates. When low salinities established an additional stress for the polychaetes they showed a response analogous to an oxyconformer model. In contrast, the common indigenous polychaete Nereis diversicolor kept its full heat dissipation down to severe hypoxia (ref.30). Comparing both polychaetes $N$. diversicolor had a more pronounced oxyregulating capacity than $M$. viridis although both animals did not follow either a clear oxyregulating or oxyconforming behaviour (ref.31).

\section{ENVIRONMENTAL MONITORING}

Questions of environmental protection gain more and more attention in recent times. Various proposals exist how to record metabolic and energetic changes in complex biological entities. Energy flow through such a system may serve as a sensitive indicator for its status independent of the existing complexity and for toxic agents acting on it. These energy flows may be monitored not only by indirect calorimetry of different fashion, but also by direct calorimetric determinations (ref.6). Up to now they are seldomly applied in this field of research but offer the chance of a non-specific detection. Although valuable on all levels of complexity, only a few selected examples shall be cited here for small terrestrial animals.

Pentachlorophenol (PCP) was - and in some countries still is - one of the most intensively and frequently used pesticides world-wide. It has a broad action spectrum for wood and crop protection by functioning against bacteria, fungi, algae, insects and molluscs (ref.11,12). Since it is a mighty uncoupler of oxydative phosphorylation, the energy usually stored in form of high energy bonds of organic compounds (e.g. ATP) is released as heat which can easily be detected by calorimetric methods. Different components of a complex forest system were investigated for their metabolism under increasing PCP concentrations. Just as litter and soil, small forest animals like wood lice (Oniscus asellus), pill bugs (Armadillidium vulgare), carabid beetles (Nebria brevicollis) or earthworms (Eisenia foetida) answered with significant metabolic stimulations. They were most easily recorded with the earthworm since it lacks the protective cuticle of the beetles. In the mentioned experiments there was a linear relationship between the PCP uptake and the increase in mass specific heat output (ref.11).

Another carabid beetle, Pterostichus oblongopunctatus, was used as a model insect for a Berlin forest system and treated with lead contaminated food and sulphure dioxide loaded air. Uptake of lead resulted in a strong metabolic increase and a more than twofold heat production rate while $\mathrm{SO}_{2}$ lowered the heat dissipation slightly as compared to the sham exposed control. A combination of both poisons gave rise to the calorimetric signal but not as much as lead alone (ref.32). In a specially developed instrument Lovrien and coworkers (ref.33) investigated the calorimetric answers of cabbage looper larvae (Trichoplusia ni) to microgram amounts of benzene and aliphatic hydrocarbons in the air flow through the calorimeter. The authors found strong exothermic irritations in the case of benzene and much smaller ones for the hydrocarbons.

\section{CONCLUSIONS}

The experiments cited above show that direct calorimetry - if needed in combination with different methods of indirect calorimetry - is a sensitive, quick and promising tool for biological investigations of physiological or environmental problems. Its non-specificity offers the chance to detect even unexpected effects, its non-invasiveness to reduce the stress to the experimental animals and to keep them alive for further tests. Calorimetric techniques have been highly refined in recent years. Miniaturized sensors for oxygen, $\mathrm{pH}$ and other parameters and light guides for illumination allow for sophisticated simultaneous investigations on differently sized animals. After a period of stagnation in the middle of this century, direct calorimetry is flowering again. 


\section{REFERENCES}

1.

M.Kleiber, The Fire of Life, J. Wiley \& Sons, New York (1961).

2. M.J.Dauncey, Thermochim. A, 193, 1-40 (1991).

3. R.S.Criddle, R.W.Breidenbach and L.D.Hansen. Thermochim. A. 193, 67-90 (1991).

4. J.R.Brett and T.D.D.Groves. In Fish Physiology (W.S.Hoar, D.J.Randall and J.R.Brett, eds.), vol. VIII: Bioenergetics and Growth. pp.279-352, Academic Press, New York (1979).

5. I.Lamprecht. Thermochim. A. 94, 113-122 (1985) .

6. U.Reh. Thermochim. A. 193, 107-124 (1991).

7. K.D.Löhr, P.Sayyadi and I.Lamprecht. Experientia 32, 1002-1003 (1976).

8. K.D.Löhr, P.Sayyadi and I.Lamprecht. In Thermodynamics of Biological Processes (I.Lamprecht and A.I.Zotin, eds.) pp.197-203, de Gruyter, Berlin (1978).

9. E.Schmolz and O.Schulz. Thermochim. A. 251, 241-245 (1995).

10. M.Harak, I.Lamprecht and A.Kuusik. Thermochim. A. 276, 41-47 (1996).

11. I.Lamprecht, Ch.Motzkus, B.Schaarschmidt and D.Coenen-Stass. Thermochim. A. 172, 87-94 (1990).

12. K.Drong, I.Lamprecht, Ch.Motzkus and B.Schaarschmidt. Thermochim. A. 193, $125-134$ (1991).

13. K.Drong and I.Lamprecht. Pure \& Appl. Chem. 65,9, 1967-1972 (1993).

14. I.Lamprecht. Thermochim. A. 300, (1997) in press.

15. W.Becker and I.Lamprecht. Z.f.Parasitenk. 53, 297-305 (1977).

16. I.Lamprecht and W.Becker. Thermochim. A. 130, 87-93 (1988).

17. L.Fahrenholz, I.Lamprecht and B.Schricker. J. Comp. Physiol. B 162, 119-130 (1992).

18. A.I.Zotin. Thermodynamic Aspects of Developmental Biology. Monographs in Developmental Biology, vol.5. Karger, Basel (1972).

19. A.Kuusik, M.Harak, K.Hiiesaar, L.Metspalu and U.Tartes. Thermochim. A. 251, $247-253$ (1995).

20. E.Schmolz, I.Lamprecht and B.Schricker. Apidol. 27 (1996).

21. C. Schulz, M. Thuy and G. Wegener. Thermochim. A. 187, 71-78 (1991).

22. T. Moratzky, G. Burkhardt, W. Weyel and G. Wegener. Thermochim. A. 229, 193-204 (1993).

23. H.Schmidt, A.Wichmann, I.Lamprecht and I.Zerbst-Boroffka. J. Comp. Physiol. B 166, 205-214 (1996).

24. V.J.T. van Ginneken, A. Gluvers, R. W. van der Linden, A.D.F. Addink and G.E.E.J.M. van den Thillart. Thermochim. A. 247, 209-224 (1994).

25. V.J.T. van Ginneken, J. Vanderschoot, A.D.F.Addink and G.E.E.J.M. van den Thillart. Thermochim. A. 249, 143-159 (1995).

26. V.J.T. van Ginneken, A.D.F.Addink and G.E.E.J.M. van den Thillart. Thermochim. A. 276, 7-15 (1996).

27. P. Stangel and G. Wegener. Thermochim. A. 271, 101-113 (1996).

28. A. Schneider, I. Lamprecht and M.K. Grieshaber. Mitt. Deutsche Ges. Limnol. 36, 314-319 (1992).

29. A. Schneider. Thermochim. A. 271, 31-40 (1996).

30. D. Fritzsche and J.-A. von Oertzen. Thermochim. A. 251, 1-9 (1995).

31. D. Fritzsche and J.-A. von Oertzen. Mar. Biol. 121 693-699 (1995).

32. U. Rink. Doctoral Thesis, Dept. Biology, Free University, Berlin 1991

33. R.E. Lovrien, T.J. Kurtti, R. Tsang and M. Brooks-Wallace. J. Biochem. Biophys. Methods 5, $307-318$ (1981). 